\title{
Evaluation of outcomes after management of metacarpal and phalangeal fractures of hand
}

\author{
Chaurasia D.S. ${ }^{1}$, Pandey N. ${ }^{2}$ \\ ${ }^{1}$ Dr. D. S. Chaurasia, Consultant, Orthopaedician, Dev Sudha Nursing Home Tikamgarh, ${ }^{2}$ Dr. Nirnay Pandey, PGMO \\ cum Senior Resident, Department of Orthopaedics, GMC Chhindwara, MP, India.
}

Corresponding Author: Dr. Nirnay Pandey, PGMO cum Senior Resident, Department of Orthopaedics, GMC, Chhindwara (M.P.) India. E-mail: dr.nirnay@gmail.com

\begin{abstract}
Background: Fracture and dislocation of hand most frequently encounter in orthopaedics injuries fracture of phalanges and metacarpal are approximately $10 \%$ of all fracture of skeleton system phalangeal fracture comprise $46 \%$ of hand fractures, metacarpal fractures $38 \%$ and fifth metacarpal fracture $9.7 \%$, distal phalangeal fractures are most commonly encounter fracture of the hand. The annual incidence of phalangeal fracture in hand is $1 \%$ in normal population. And these injuries account for between. $2 \%$ and $3 \%$ of all patient visiting in accident and emergency unit thumb and middle finger are most frequently injured because they extend most distally during work activity. Aim \& Objectives: To evaluate the structural and functional outcomes and the results of operative and conservative management of metacarpal and phalangeal fractures of hand. Methods: The present study comprises of 62 patients of closed fractures of metacarpal and phalanx treated in the Department of Orthopaedic Surgery, Shyam Shah Medical College and associated Sanjay Gandhi Memorial Hospital Rewa (M.P.) in a span of two years. Evaluation of outcomes after management of metacarpal and phalangeal fractures of hand was done. Results: We achieved $24(85.7 \%)$ excellent and good results in conservative method for metacarpal fractures and $10(83.33 \%)$ operative methods. In Phalangeal fractures $09(90.0 \%)$ excellent and good results were achieved in conservative method and $10(83.34 \%)$ in operative methods. Excellent and good grip strength was noted in $53(85.33 \%)$ cases. Single digit involvement had better TAM then multiple digits involvement. Poor results were noted in metacarpal fractures in $04(14 \%)$ in conservative series and $02(16.0 \%)$ in operative series. Poor results were seen in phalangeal fractures in $01(10 \%)$ in conservative series and $02(16.66 \%)$ in operative series. Most common complication was finger stiffness in conservative method and pin site infection in operative methods. Conclusion: Both conservative and operative methods (surgical stabilization) for metacarpal and phalangeal fractures of hand gave good functional outcomes, depending on the fracture pattern for treatment option, single digit involvement are important determinants to achieve a better grade of total active range of motion.
\end{abstract}

Keywords: Conservative method, Metacarpal fractures, Operative methods, Phalangeal fractures, Surgical stabilization

\section{Introduction}

The special development of the human hand is largely responsible for the great handicraft of man. The hand is an organ of grasp as well as an organ of sensation and expression. By the use of our hands we learn shape, size and texture of objects and then combine this information with impressions from other senses to build in our brains the knowledge of our environment.

We use our hands not only as tools for grasping, pinching and pushing, but with our brains directing the hands we manufacture tools for special purposes. Our

Manuscript Received: $10^{\text {th }}$ August 2019

Reviewed: $20^{\text {th }}$ August 2019

Author Corrected: $28^{\text {th }}$ August 2019

Accepted for Publication: $2^{\text {nd }}$ September 2019 hands become extensions of the intellect, because by hand movements the dumb converse, with the specialized fingertips the blind read; and through the written word we learn from the past and transmit to the future. The normal hand is a pentadactylate mechanism of basic design; its finer motions and sensibility have been developed over the ages on the primitive amphibian pattern $[1,2]$.

Although the hand is compound mainly of tough material. It also includes, exact machinery of much retirement and tissue of great delicacy and specialization, such a mechanism is readily wrecked by trauma and infections and it is little wonder that hands 


\section{Original Research Article}

mangled by traumas.Mal formation, Injury and infection swell the overcoming stream of crippled handy needing repair. Surgical reconstructions of the hand require special careful tech use to minimize adhesion formation which is so prove to bind together the nicely adjusted moveable parts.

It is composite problem requiring the correlation of the various specialties' orthopedics plastic and neurosurgery. The knowledge of any one of which alone is inadequate for repairing the hand. Trauma involves all types of tissue irrespective of the artificial division of our specialties. Usually in the same traumatized limbs with flexion contracture, injury to tendons, bone joints and never we must combine plastic, orthopedic and neurologic surgery. As the problem is composite the surgeon must also be [3].

The goal with injuries to the hand is a rapid and accurate initial evaluation. In other words, once an injury occurs, the doctor strives to begin medical treatment quickly so the short- and long-term effects can be minimized. Hand injuries account for nearly $10 \%$ of hospital Emergency Department visits. A recent series of 1,000 consecutive hand injuries showed the following distribution: $42 \%$ lacerations (cuts), $27 \%$ contusions (bruises), 17\% fractures (broken bones), and $5 \%$ infections [4].

\section{Materials and Methods}

Study Design/Type of study - Prospective, randomized, single blinded study.

Sample size \& Duration of study- The present study comprises of 62 patients of closed fractures of metacarpal and phalanx treated in the Department of Orthopaedic Surgery, Shyam Shah Medical College and associated Sanjay Gandhi Memorial Hospital Rewa (M.P.) in a span of two years.

The present study group included 62 patients of closed metacarpals and phalangeal fracture with total 52 metacarpals and 30 phalanx fracture with 10 metacarpal base, 14 metacarpal neck, 24 metacarpal shaft, 4 intraarticular fracture, with 14 shaft phalanx, 6 phalanx base, 06 phalanx neck, 04 intraarticular phalanx fracture.

The single digit involved in fifty patients, two digits involved in four patients and more then three digit involved in two patients.

\section{Inclusion criteria}

1. All age patient included in the present study

2. Both intra articular and extraarticular fractures included

3. Both sex male and female

4. Multiple fracture

\section{Exclusion criteria}

1. Associated injury to upper limb

2. Open fractures

3. Pathological fracture

4. Finger with amputated digits

\section{Radiological assessment}

- Brewertan view to reveals fractures pattern involving metacarpal bases.

- Mehara view delineates anatomy around the base of the second metacarpal and the trapezoid.

- Sky line view for vertical impactions fracture in metacarpal head

- Modified lateral views: Modified lateral views of the metacarpals are sometimes necessary because little of the shaft or head can be observed on a true lateral radiograph of the hand. To study the index and middle finger, the hand should be pronated $30^{\circ}$ from the lateral. To study the ring and small fingers, the hand should be supinated $30^{\circ}$ from the lateral. 
Original Research Article

Table 1: Salter-Harris fracture classification in peadiatrics.

\begin{tabular}{|l|l|}
\hline Type I & Separation of the epiphysis from the metaphysic with a shear mechanism \\
\hline Type II & $\begin{array}{l}\text { Account for } 80 \% \text { of pediatric hand fractures, Separation of the epiphysis with a fracture } \\
\text { through the metaphysic }\end{array}$ \\
\hline Type III & $\begin{array}{l}\text { i. Intra-articular fracture through the epiphysis without epiphyseal plate involvement } \\
\text { ii. Poor prognosis unless well reduced }\end{array}$ \\
\hline Type IV & $\begin{array}{l}\text { i. Fracture through the epiphysis that extends through the epiphyseal plate and metaphysic } \\
\text { ii. Poor prognosis without anatomic reduction }\end{array}$ \\
\hline Type V & $\begin{array}{l}\text { i. Compression fracture with damage to the epiphyseal plate } \\
\text { ii. Poor prognosis, with potential for growth arrest }\end{array}$ \\
\hline
\end{tabular}

Data analysis: The various statistical techniques i.e. the mean, standard deviation and test of significance (t-test and chisquare-test) were used for drawing valid conclusions. Statistical analysis done using student t-test. SPSS 13.0 software was used to calculate $\mathrm{p}$ value.

Surgical Procedures done: We treated fractures by both conservative and operative method.

Conservative method- It is used for undisplaced and satisfactory acceptable position of fracture. We used slab, cast, strapping, splints for immobilization of fracture.

\section{Operative method-Skeletal Stablization}

[a] K-wire: K-wires and external fixators we find best methods for fixation

\section{[b] External Fixator}

Follow up: Every case were subjected to attend the Orthopaedic O.P.D. for follow-up and patients were examined for the range of motion, active and passive grip strength and developing complications and advised physiotherapy accordingly. There after patient were reviewed on 1stweek, 3rd week 6th week, 3rd month, 6th month and one year post operatively.

Assessment of result- TAM and grip strength used for assessment as suggested by American Society for surgery of hand. At final follow-up total active motion(TAM)active flexion of metacarpophalangeal, proximal inter phalangeal and distal interphalangeal joint minus the extension deficit in these joint was use to assess the range of motion. The range of motion at distal interphalangeal joint (DIP-ROM) was also documented these two grading system were use to assess the result.

Table 2: Two grading system used to assess range of motion at final follow-up.

\begin{tabular}{|c|c|c|}
\hline Grade & TAM (normal-260) & DIP ROM (normal-70) \\
\hline Excellent & 240 or greater & 60 or greater \\
\hline Good & $210-239$ & $40-59$ \\
\hline Fair & $180-209$ & $20-39$ \\
\hline Poor & less then 180 & less then 20 \\
\hline
\end{tabular}

\section{Functional assessment}

1. Evaluation of principal function of hand

2. Pinch grip

3. Thumb to side index grip

4. Grasp

5. Grip strength 
Original Research Article

Table 3: Grip strength.

\begin{tabular}{|c|c|c|}
\hline S. No. & Grip strength & Percentage (\%) \\
\hline 1 & Excellent & $90-100 \%$ \\
\hline 2 & Good & $70-90 \%$ \\
\hline 3 & Poor & $<70 \%$ \\
\hline
\end{tabular}

\section{Results}

We had studied 62 cases of metacarpal and phalangeal fractures of hands treated either by conservative or operative method.

Table-4: Mode of trauma.

\begin{tabular}{|c|c|c|c|}
\hline S. No. & Trauma & No. of Patients & Percentage \\
\hline 1 & RTA & 26 & $41.93 \%$ \\
\hline 2 & Assault & 16 & $25.88 \%$ \\
\hline 3 & Agricultural & 08 & $12.90 \%$ \\
\hline 4 & Machinery Injury & 04 & $6.45 \%$ \\
\hline 5 & Fall of Object & 08 & $12.90 \%$ \\
\hline \multicolumn{2}{|c|}{ Total } & $\mathbf{6 2}$ & $\mathbf{1 0 0 . 0} \%$ \\
\hline
\end{tabular}

Mode of trauma in maximum number of cases was RTA, followed by assault. $64.52 \%$ metacarpal fractures were more common.

Table-5: Metacarpal fracture.

\begin{tabular}{|c|c|c|c|c|c|}
\hline S. No. & \multicolumn{2}{|c|}{ Site of Fracture } & No. of Patients & No. of Fractures & Percentage \\
\hline 1 & & Neck & 10 & 14 & $25 \%$ \\
\hline \multirow{4}{*}{2} & \multirow{4}{*}{ Shaft } & Oblique & 06 & 08 & $15 \%$ \\
\hline & & Spiral & 00 & 00 & - \\
\hline & & Transverse & 08 & 12 & $20 \%$ \\
\hline & & Comminuted & 04 & 04 & $10 \%$ \\
\hline 3 & \multicolumn{2}{|l|}{ Base } & 08 & 10 & $20 \%$ \\
\hline 4 & \multicolumn{2}{|c|}{ Intra-articular } & 04 & 04 & $10 \%$ \\
\hline \multicolumn{3}{|c|}{ Total } & 40 & 52 & $100.0 \%$ \\
\hline
\end{tabular}

Maximum involvement was of shaft of metacarpal (45\%) followed by neck of metacarpal (25\%). Minimal involvement was of intraarticular fractures $(10.0 \%)$.

Table-6: Affection of digit in phalangeal fracture.

\begin{tabular}{|c|c|c|c|c|}
\hline S. No. & $\begin{array}{c}\text { Digit Affected in Phalanx } \\
\text { Fracture }\end{array}$ & No. of Patients & No. of Digit & Percentage \\
\hline 1 & Thumb & 04 & 06 & $20 \%$ \\
\hline 2 & Middle Finger & 04 & 06 & $20 \%$ \\
\hline 3 & Ring Finger & 03 & 05 & $16.66 \%$ \\
\hline 4 & Little Finger & 07 & 09 & $30 \%$ \\
\hline 5 & Index Finger & 04 & 04 & $13.33 \%$ \\
\hline \multicolumn{2}{|r|}{ Total } & 22 & 30 & $100.0 \%$ \\
\hline
\end{tabular}


Original Research Article

Table-7: Type of phalangeal fracture.

\begin{tabular}{|c|c|c|c|c|}
\hline S. No. & Type & No. of Patients & No. of Fractures & Percentage \\
\hline 1 & Transverse & 10 & 14 & $46.66 \%$ \\
\hline 2 & Oblique & 06 & 06 & $20.0 \%$ \\
\hline 3 & Spiral & - & - & - \\
\hline 4 & Comminuted & 04 & 06 & $20.0 \%$ \\
\hline 5 & Intra articular & 02 & 04 & $13.33 \%$ \\
\hline \multicolumn{2}{|r|}{ Total } & 22 & 30 & $100.0 \%$ \\
\hline
\end{tabular}

Transverse (46.66\%) were more common. Maximum involvement were occurs in shaft of phalanx (46.665) and minimal were occurred intraarticular type (13.33\%).70\% patients treated conservatively and $30 \%$ with operative procedure.

Table 8: Method of operative procedure in metacarpal fracture.

\begin{tabular}{|c|l|l|c|c|}
\hline \multirow{2}{*}{ S. No. } & \multicolumn{1}{|c|}{ Methods } & No. of Patients & Percentage \\
\hline \multirow{2}{*}{1} & \multirow{2}{*}{ K-wire } & Straight K-wire & 06 & $50.0 \%$ \\
\cline { 3 - 4 } & & Cross K-wire & 03 & $25.0 \%$ \\
\cline { 3 - 5 } & & Transverse K-wire & 01 & $8.33 \%$ \\
\hline 2 & External Fixator & 01 & $8.33 \%$ \\
\hline 3 & Encerclage & 01 & $8.34 \%$ \\
\hline \multicolumn{2}{|c|}{ Total } & $\mathbf{1 2}$ & $\mathbf{1 0 0 . 0 \%}$ \\
\hline
\end{tabular}

Table-9: Method of operative procedure in metacarpal fracture.

\begin{tabular}{|c|c|l|c|c|}
\hline \multirow{2}{*}{ S. No. } & \multicolumn{1}{|c|}{ Methods } & No. of Patients & Percentage \\
\hline \multirow{2}{*}{1} & \multirow{2}{*}{ K-wire } & Straight K-wire & 08 & $66.67 \%$ \\
\cline { 3 - 5 } & & Cross K-wire & 02 & $16.66 \%$ \\
\hline 2 & JESS & 02 & $16.67 \%$ \\
\hline \multicolumn{2}{|c|}{ Total } & $\mathbf{1 2}$ & $\mathbf{1 0 0 . 0 \%}$ \\
\hline
\end{tabular}

Table-10: Metacarpal fracture.

\begin{tabular}{|c|c|c|c|c|c|}
\hline \multirow{2}{*}{ S. No. } & \multirow{2}{*}{ Result } & \multicolumn{2}{|c|}{ Conservative } & \multicolumn{2}{c|}{ Operative } \\
\cline { 2 - 6 } & & No & $\mathbf{\%}$ & No. & $\%$ \\
\hline 1 & Excellent & 10 & 35.71 & 04 & 33.33 \\
\hline 2 & Good & 14 & 50.0 & 06 & 50.0 \\
\hline 3 & Poor & 04 & 14.0 & 02 & 16.0 \\
\hline \multicolumn{2}{|c|}{ Total } & $\mathbf{2 8}$ & $\mathbf{1 0 0 . 0}$ & $\mathbf{1 2}$ & $\mathbf{1 0 0 . 0}$ \\
\hline
\end{tabular}

we achieved $85.71 \%$ excellent and good result in conservative method and $83.33 \%$ in operative method.

Table-11: Phalangeal fracture.

\begin{tabular}{|c|c|c|c|c|c|}
\hline \multirow{2}{*}{ S. No. } & \multirow{2}{*}{ Result } & \multicolumn{2}{|c|}{ Conservative } & \multicolumn{2}{c|}{ Operative } \\
\cline { 2 - 5 } & & No & \% & No. & \% \\
\hline 1 & Excellent & 03 & 30.0 & 04 & 33.34 \\
\hline 2 & Good & 06 & 60.0 & 06 & 50.0 \\
\hline 3 & Poor & 01 & 10.0 & 02 & 16.66 \\
\hline \multicolumn{2}{|c|}{ Total } & $\mathbf{1 0}$ & $\mathbf{1 0 0 . 0}$ & $\mathbf{1 2}$ & $\mathbf{1 0 0 . 0}$ \\
\hline
\end{tabular}

we achieved $90 \%$ excellent and good result in conservative method and $83.34 \%$ in operative method. 
Original Research Article

Table-12: Grip strength.

\begin{tabular}{|c|c|c|c|}
\hline S. No. & Grip Strength & No. of Patients & Percentage \\
\hline 1 & Excellent $(90-100 \%)$ & 21 & $33.87 \%$ \\
\hline 2 & Good $(70-90 \%)$ & 32 & $51.16 \%$ \\
\hline 3 & Poor $(<70 \%)$ & 09 & $14.51 \%$ \\
\hline \multicolumn{2}{|r|}{ Total } & $\mathbf{6 2}$ & $\mathbf{1 0 0 . 0 \%}$ \\
\hline
\end{tabular}

Table-13: Complications.

\begin{tabular}{|c|c|c|c|c|}
\hline S. No. & Complications & Conservative & Operative & Total \\
\hline 1 & Finger stiffness & 08 & 03 & 11 \\
\hline 2 & Pain & 07 & 05 & 12 \\
\hline 3 & Pin site infection & NA & 03 & 03 \\
\hline 4 & Residual deformity & 05 & 04 & 09 \\
\hline 5 & Sudeck osteodystrophy & 01 & NIL & 01 \\
\hline 6 & Nerve Palsy & NIL & NIL & NIL \\
\hline 7 & Mal-union & 02 & NIL & 02 \\
\hline
\end{tabular}

Most common complication in conservative series was finger stiffness, while prime position in operative series was taken by pin site infection. The most common complication in whole series was clinically in considerable pain.

$28(70.0 \%)$ patients were treated with conservative and $12(30.0 \%)$ by operative methods. K-wire were the most commonly used material for fixation of fractures $10(83.33 \%)$ in metacarpals and $10(73.33 \%)$ in phalangeal fractures. External fixator (JESS) was used in $01(8.33 \%)$ in metacarpal and $02(16.67 \%)$ in phalangeal fractures. Most fractures were immobilized for 3-4 weeks in conservative method.

We achieved $24(85.7 \%)$ excellent and good results in conservative method for metacarpal fractures and $10(83.33 \%)$ operative methods. In Phalangeal fractures 09 (90.0\%) excellent and good results were achieved in conservative method and $10(83.34 \%)$ in operative methods. Excellent and good grip strength was noted in $53(85.33 \%)$ cases. Single digit involvement had better TAM then multiple digits involvement.

Poor results were noted in metacarpal fractures in $04(14 \%)$ in conservative series and $02(16.0 \%)$ in operative series. Poor results were seen in phalangeal fractures in $01(10 \%)$ in conservative series and $02(16.66 \%)$ in operative series. Most common complication was finger stiffness in conservative method and pin site infection in operative methods.

\section{Discussion}

In Department of Orthopaedic Surgery, Shyam Shah Medical College and associated Sanjay Gandhi Memorial Hospital, Rewa (M.P.),62 patients with 82 metacarpal and phalangeal fracture were treated either by operative or conservative methods, during the span of two years.

Gupta R et al did a prospective study for evaluation of surgical stabilization of metacarpal and phalangeal fractures of hand. Overall results were excellent to good in $87 \%$. Better total active range of motion (excellent grade) was observed in metacarpal fractures (47\%) versus phalanx fractures (31\%); closed fractures $(57 \%)$ versus open fractures $(27 \%)$; and single digit involvement (55\%) versus multiple digits (29\%). Excellent total active range of motion was observed with all four plate and screw/ screw fixation technique $(100 \%)$ and closed reduction and percutaneous kirschner wire fixation (60\%). Surgical stabilization of metacarpal and phalangeal fractures of hand seems to give good functional outcome. Closed fractures and fractures with single digit involvement have shown a better grade of total active range of motion [1].

Duncan RW et al did an analysis of the recovery of active motion and of complications of open hand fractures. Results, evaluated on the basis of total active range of digital motion achieved at final follow-up, 


\section{Original Research Article}

correlated highly with severity of soft tissue injury. When open fractures of comparable severity were contrasted between groups that did and did not require additional extension by incision to achieve acceptable reduction and stabilization, there was some additional loss of active range of motion in the surgically treated group. Metacarpal fractures had significantly better outcomes than phalangeal fractures. Fractures involving the proximal phalanx or the proximal interphalangeal joint had the poorest prognosis, especially when they were associated with tendon injury. There were significant complications in 13 fingers. Infection and late amputation were related to wound severity [2].

Trevisan $\mathrm{C}$ et al did a lot of work on low-severity metacarpal and phalangeal fractures treated with miniature plates and screws. They retrospectively reviewed 44 patients of a consecutive series with 56 low-severity metacarpal and/or phalangeal fractures. These very favourable results suggest that miniature plates and screws are a possible choice in the treatment of these fractures.

Kodama $\mathrm{N}$ et al Evaluated the outcomes of operative treatment for metacarpal and phalangeal fractures in athletes returning early to play and discussed the more effective methods that permit rapid early return to athletic activity. They concluded that an early comeback to training and competition can be permitted exclusively for patients with metacarpal and phalangeal fractures. It is important for the attending physician to administer such treatment after obtaining informed consent and develop a trusting relationship with the patient and other related individuals while paying attention to their hope of quick recovery $[3,4]$.

Tavassoli LC et al retrospectively evaluated three methods of closed treatment; specifically, we investigated whether the position of immobilization of the metacarpophalangeal joint or the absence of a range of motion of the interphalangeal joints affected the short-term outcome or fracture alignment.

When immobilization was discontinued by five weeks, the position of the metacarpophalangeal joints and the absence or presence of interphalangeal joint motion during the immobilization had little effect on motion, grip strength, or fracture alignment. This finding contradicts the conventional teaching that the metacarpophalangeal joint must be immobilized in flexion to prevent long-term loss of joint extension. Patient comfort, ease of application, and the surgeon's familiarity with the technique should influence the choice of immobilization [5].
Kawamura $\mathrm{K}$ et al evaluated fixation choices for closed simple unstable oblique phalangeal and metacarpal fractures. Similar study was done by Horton TC, Hatton $\mathrm{M}$ et al. They did a prospective randomized controlled study and compared fixation of long oblique and spiral shaft fractures of the proximal phalanx by closed reduction and percutaneous Kirschner wiring versus open reduction and lag screw fixation. There was no significant difference in the functional recovery rates or in the pain scores for the two groups. X-rays showed similar rates of malunion and there were no statistically significant differences in range of movement or grip strength $[6,7]$.

Operative treatment of metacarpal and phalangeal shaft fractures was also done by Kozin $\mathrm{SH}$ et al. They concluded that nonoperative treatment is indicated for reducible and stable fracture configurations. Irreducible or unstable fracture patterns require open or closed reduction and fixation. Reduction must be assessed in flexion and extension to ensure correct rotatory alignment. Fracture fixation can be achieved with the use of Kirschner wires, interfragmentary screws, or plates. The outcome after surgery is greatly influenced by the condition of the surrounding soft tissues; therefore, surgical trauma should be minimized to optimize the result [8].

The purpose of the study by Omokawa S, Fujitani R et al was to prospectively evaluate clinical results for open reduction and internal fixation of unstable metaphyseal fractures of the metacarpal and phalangeal bones using a miniature titanium plate. Bone union was successfully achieved in all patients over an average period of 2.6 months. The final range of total active motion (\%TAM) was excellent $(>85 \%)$ for 26 , good $(70 \%-84 \%)$ for 17 , fair $(50 \%-69 \%)$ for 5 , and poor $(<49 \%)$ for 3 . They concluded that low-profile titanium plate system was highly effective in maintaining anatomic reduction. The postoperative complication rate was relatively low, and the objective outcomes approached a reasonable level at 1-year follow-up [9].

Shimizu $\mathrm{T}$ et al studied predictors of the postoperative range of finger motion for comminuted periarticular metacarpal and phalangeal fractures treated with a titanium plate. The purpose of this prospective cohort study was to identify independent prognostic factors of the postoperative total active motion (\%TAM) in the treatment of metacarpal and phalangeal fractures. Univariate and multivariate linear regression analysis were used to identify the degree to which variables affect $\%$ TAM at the final follow-up. Phalangeal 


\section{Original Research Article}

fracture, increasing age and associated soft-tissue injury were important risk factors to identify the postoperative $\%$ TAM in the treatment of comminuted periarticular metacarpal or phalangeal fracture with a titanium plate [10].

Ozer K, Gillani S et al did comparison of clinical and radiographic outcomes of intramedullary nailing versus plate-screw fixation of extra-articular metacarpal fractures. There were no significant differences in the clinical outcomes using either technique. Although operative time was shorter in the IMN group than in the PS group, the incidences of loss of reduction, penetration to the metacarpal-phalangeal joint, and secondary surgeries for hardware removal in the operating room were much higher in the IMN group [11].

Orbay JL et al studied the treatment of unstable metacarpal and phalangeal shaft fractures with flexible nonlocking and locking intramedullary nails. Similar study was done by Gonzalez $\mathrm{MH}$ et al who studied flexible intramedullary nailing for metacarpal fractures. The average follow-up time was 9 months (range, 2 to 34 months) with 15 patients lost to follow-up examination. All fractures went on to heal. Three complications occurred: backing out of a rod in one case and bending of the rods after repeat trauma in two. There were no infections. Flexible intramedullary nailing of specific metacarpal fractures affords excellent results with a low complication rate. Proper selection of fractures and good surgical technique are necessary to avoid complications $[12,13]$.

Wong TC et al did comparison between percutaneous transverse fixation and intramedullary $\mathrm{K}$-wires in treating closed fractures of the metacarpal neck of the little finger. They reviewed at a mean follow-up of 24 months for radiological and functional outcome in terms of union rate, union time, complication rate, pain, movement and grip strength. Radiographs did not show any significant differences in the union rate and union time. There was no statistical difference in complication rate, pain scores, total active motion and grip strength between the two groups. The authors concluded that both methods are comparable, good and safe methods of treating closed, displaced fractures of the metacarpal neck of the little finger, without significant complication [14].

Faraj AA et al did percutaneous intramedullary fixation of metacarpal shaft fractures. Twenty-two displaced metacarpal shaft fractures in 19 patients were stabilized with multiple intramedullary K-wires. These were inserted percutaneously through a small window in the base of the metacarpal and were buried in the bone. All the fractures that we were able to follow-up united.

Westbrook AP et al did work on the clinical significance of malunion of fractures of the neck and shaft of the little finger metacarpal. Outcome measures included the grip strength of the little finger and the DASH score. Fracture malunion was assessed by clinical measurement of little finger ray shortening and measurement of palmar angulation. There were no differences between the outcomes of the 18 metacarpal neck fractures treated operatively and the 105 treated non-operatively. The DASH score $(P=0.001)$ and aesthetic outcome $(P=0.013)$ were significantly better for the 113 metacarpal shaft fractures treated nonoperatively than for the 26 treated operatively $[15,16]$.

Strub B, Schindele $\mathrm{S}$ did a prospective study on intramedullary splinting or conservative treatment for displaced fractures of the little finger metacarpal neck. A radiological and clinical assessment of flexion and extension of the small finger metacarpophalangeal joint was done at 2 and 6 weeks, and at 3, 6 and 12 months. In addition, patient satisfaction and grip strength were recorded at 12 months.

No statistically significant differences in range of motion and grip strength were found between the two groups. Patient satisfaction and the appearance were superior in the surgically treated group. It was concluded that intramedullary splinting for displaced fractures of the little finger metacarpal neck offers an aesthetic, but not a functional advantage [17].

Freeland $\mathrm{AE}$ et al in their review of new developments on extraarticular hand fractures in adults confirmed that small amounts of metacarpal shortening or dorsal angulation cause minimal functional impairment. Unilateral excision of the lateral band and oblique fibers of the extensor apparatus of the metacarpophalangeal joint facilitates proximal phalangeal fracture exposure and may improve functional recovery.

Results using open mini screw fixation of oblique extraarticular metacarpal and phalangeal fractures may be comparable to those of percutaneous Kirschner wire fixation. Bicortical self-tapping mini screw fixation of extra-articular oblique metacarpal and phalangeal fractures simplifies screw insertion and provides stability comparable to that of fractures fixed with lag screws. Percutaneous intramedullary wire fixation may 
Original Research Article

afford suitable fixation for unstable extra-articular oblique as well as transverse metacarpal fractures. Locked intramedullary nails may offer similar advantages. Unicortical screw fixation of mini plates securing transverse extra-articular metacarpal fractures affords stability comparable to that of bicortical screw fixation while creating less bone damage.

The dissection required for plate fixation and the small surface area of transverse fractures delay and occasionally impair bone healing. Primary bone grafting of diaphyseal defects in clean stable wounds may shorten and simplify treatment and decrease morbidity.

As little as $1.7 \mathrm{~mm}$ of flexor tendon excursion during the first 4 weeks after reduction or repair may substantially diminish peritendonous adhesions at the fracture site.

Synchronous wrist and digital exercises may also reduce peritendonous fracture adhesions. Early motion of adjacent joints in closed simple metacarpal fractures expedites recovery of motion and strength without adversely affecting fracture alignment and leads to earlier return to work [18].

\section{Limitation of the present study}

1. Small sample size

2. Chances of bias

3. Single center trial

\section{Conclusion}

At the end of the study we concluded that --

- Both conservative and operative methods (surgical stabilization) for metacarpal and phalangeal fractures of hand gave good functional outcomes, depending on the fracture pattern for treatment option, single digit involvement are important determinants to achieve a better grade of total active range of motion.

- There was equal effectiveness of both operative and conservative treatment of metacarpal and phalangeal fractures. The better anatomical outcome in terms of the degree of angular deformity did not translate directly into the better function of hand.

- Operative management of metacarpal and phalangeal fractures had advantage over the close method of treatment especially in displaced, unstable, comminuted, intra-articular and multiple fractures.

- Complication should be minimized by precision in selection of cases for operative treatment.

\section{What this study adds to the existing knowledge?}

Indiscriminate use of operative fixation especially in fractures which are stable, un-displaced/minimally displaced and which can be managed well conservatively should be avoided. The joints should be mobilized early following fixation to prevent stiffness of fingers and to regain the desired range the motion of the joint. Early postoperative physiotherapy of the hand is a must. Finally, it may be aspired that with more experience, better facilities, perfection of techniques and more ready availability of microsurgery excellentfunctional and cosmetic results will be achieved.

\section{Contribution by Different Authors}

Dr. D. S. Chaurasia: Concept and design of the study

Dr. Nirnay Pandey: Data collection and references

Conflict of interest: None declared.

Funding: Nil, Permission from IRB: Yes

Ethical approval: Taken

\section{References}

1. Gupta R, Singh R, Siwach RC, Sangwan SS, Magu NK, Diwan R. Evaluation of surgical stabilization of metacarpal and phalangeal fractures of hand. Ind $\mathrm{J}$ Orthopaed. 2007; 41(3):224. doi: 10.4103/0019-5413. 33687

2. Duncan RW, Freeland AE, Jabaley ME, Meydrech EF. Open hand fractures: an analysis of the recovery of active motion and of complications. J Hand Surg Am. 1993; 18(3): 387-394. doi:10.1016/0363-5023(93) 90080-M

3. Trevisan C, Morganti A, Casiraghi A, Marinoni EC. Low-severity metacarpal and phalangeal fractures treated with miniature plates and screws. Arch Orthop Trauma Surg. 2004;124(10):675-680.Epub 2004 Oct 28. doi: $10.1007 / \mathrm{s} 00402-004-0745-7$

4. Kodama N, Takemura Y, Ueba H, Imai S, Matsusue Y. Operative treatment of metacarpal and phalangeal fractures in athletes: early return to play. J Orthop Sci. 2014; 19(5): 729-736. doi: 10.1007/s00776-0140584-5. Epub 2014 Jun 10.

5. Tavassoli J, Ruland RT, Hogan CJ, Cannon DL. Three cast techniques for the treatment of extra-articular metacarpal fractures. Comparison of short-term outcomes and final fracture alignments. J Bone Joint Surg Am. 2005; 87(10): 2196-2201. doi:10.2106/JBJS. D.03038 


\section{Original Research Article}

6. Kawamura K, Chung KC. Fixation choices for closed simple unstable oblique phalangeal and metacarpal fractures. Hand Clin. 2006;22(3):287-295.doi:10. 1016 /j. hcl. 2006.02.018

7. Horton TC, Hatton M, Davis TR. A prospective randomized controlled study of fixation of long oblique and spiral shaft fractures of the proximal phalanx: closed reduction and percutaneous Kirschner wiring versus open reduction and lag screw fixation. J Hand Surg Br. 2003;28(1):5-9. doi: https://doi.org/10. 1054/ jhsb. 2002.0807

8. Kozin SH, Thoder JJ, Lieberman G. Operative treatment of metacarpal and phalangeal shaft fractures. J Am Acad Orthop Surg. 2000;8(2):111-121.

9. Omokawa S, Fujitani R, Dohi Y, Okawa T, Yajima H. Prospective outcomes of comminuted periarticular metacarpal and phalangeal fractures treated using a titanium plate system. J Hand Surg Am. 2008;33 (6):857-863. doi: 10.1016/j.jhsa.2008.01.040.

10. Shimizu T, Omokawa S, Akahane M, Murata K, Nakano K, Kawamura K, et al. Predictors of the postoperative range of finger motion for comminuted periarticular metacarpal and phalangeal fractures treated with a titanium plate. Injury. 2012;43(6):940-945. doi: 10. 1016/j. injury. 2012. 02.011. Epub 2012 Mar 28.

11. Ozer K, Gillani S, Williams A, Peterson SL, Morgan S. Comparison of intramedullary nailing versus plate-screw fixation of extra-articular metacarpal fractures. J Hand Surg Am. 2008;33(10):1724-1731. doi: 10.1016/j.jhsa.2008.07.011.
12. Orbay JL, Touhami A. The treatment of unstable metacarpal and phalangeal shaft fractures with flexible nonlocking and locking intramedullary nails. Hand Clin. 2006; 22(3):279-286. doi:10.1016/j.hcl. 2006.02. 017

13.Gonzalez MH, Igram CM, Hall RF Jr. Flexible intramedullary nailing for metacarpal fractures. J Hand Surg Am. 1995;20(3):382-387. doi:10.1016/S0363-5023 (05) 80091-7

14. Wong TC, Ip FK, Yeung SH. Comparison between percutaneous transverse fixation and intramedullary Kwires in treating closed fractures of the metacarpal neck of the little finger. J Hand Surg Br. 2006;31(1):61-65. Epub 2005 Aug 31. doi:10.1016/j.jhsb.2005.06.022

15. Faraj AA, Davis TR. Percutaneous intramedullary fixation of metacarpal shaft fractures. J Hand Surg Br. 1999;24(1):76-79.

16. Westbrook AP, Davis TR, Armstrong D, Burke FD. The clinical significance of malunion of fractures of the neck and shaft of the little finger metacarpal. J Hand Surg Eur Vol. 2008; 33(6):732-739. doi: 10.1177/1753 193408092497. Epub 2008 Oct 20.

17. Strub B, Schindele S, Sonderegger J, Sproedt J, von Campe A, Gruenert JG. Intramedullary splinting or conservative treatment for displaced fractures of the little finger metacarpal neck? A prospective study. J Hand Surg Eur Vol. 2010;35(9):725-729. doi: 10.1177/ 1753193410377845. Epub 2010 Jul 21.

18.Freeland AE, Orbay JL. Extraarticular hand fractures in adults: a review of new developments. Clin Orthop Relat Res. 2006;445:133-145. doi:10.1097/01. blo.000 0205888.04200.c5

\section{How to cite this article?}

Chaurasia D.S, Pandey N. Evaluation of outcomes after management of metacarpal and phalangeal fractures of hand. Surgical Update: Int J surg Orthopedics.2019;5(4):271-280.doi:10.17511/ijoso.2019.i04.07 